\title{
Acknowledgement to Reviewers of the International Journal of Financial Studies in 2016
}

\author{
International Journal of Financial Studies Editorial Office \\ Published: 11 January 2017 \\ MDPI AG, St. Alban-Anlage 66, 4052 Basel, Switzerland; ijfs@mdpi.com
}

The editors of the International Journal of Financial Studies would like to express their sincere gratitude to the following reviewers for assessing manuscripts in 2016.

We greatly appreciate the contribution of expert reviewers, which is crucial to the journal's editorial process. We aim to recognize reviewer contributions through several mechanisms, of which the annual publication of reviewer names is one. Reviewers receive a voucher entitling them to a discount on their next MDPI publication and can download a certificate of recognition directly from our submission system. Additionally, reviewers can sign up to the service Publons (https://publons.com) to receive recognition. Of course, in these initiatives we are careful not to compromise reviewer confidentiality. Many reviewers see their work as a voluntary and often unseen part of their role as researchers. We are grateful to the time reviewers donate to our journals and the contribution they make.

If you are interested in becoming a reviewer for the International Journal of Financial Studies, see the link at the bottom of the webpage http://www.mdpi.com/reviewers.

The following reviewed for the International Journal of Financial Studies in 2016:

$\begin{array}{lll}\text { Agliardi, Elettra } & \text { Dagoumas, Athanasios } & \text { He, Kaijian } \\ \text { Aid, René } & \text { Das, Kuntal } & \text { Hegerty, Scott William } \\ \text { Alexandridis, Antonis } & \text { Dayanandan, Ajit } & \text { Hua, Xiuping } \\ \text { Al-Zoubi, Haitham } & \text { D'Ecclesia, Rita } & \text { Hung, Jui-cheng } \\ \text { Andraz, Jorge M. } & \text { Deng, Ai } & \text { Ivanov, Stoyu I. } \\ \text { Antzoulatos, Angelos A. } & \text { Dermine, Jean } & \text { Jain, Anil K. } \\ \text { Ari, Ayşe } & \text { Di Giacomo, Marina } & \text { Jansson, Magnus } \\ \text { Atukeren, Erdal } & \text { Dionne, Georges } & \text { Karoglou, Michail } \\ \text { Barro, Diana } & \text { Donadelli, Michael } & \text { Kinateder, Harald } \\ \text { Barroso, Pedro } & \text { Donker, Han } & \text { Koblyakova, Alla } \\ \text { Barth, James } & \text { Dyakov, Teodor. C. } & \text { Kocenda, Ezven } \\ \text { Bee, Marco } & \text { Ender, Manuela } & \text { Koutmos, Dimitrios } \\ \text { Belomestny, Denis } & \text { Finco, Adele } & \text { Kudryavtsev, Andrey } \\ \text { Berge, Travis J. } & \text { Gabillon, Emmanuelle } & \text { Lamb, Reinhold P. } \\ \text { Bergstresser, Daniel } & \text { Galil, Koresh } & \text { Leung, Tim } \\ \text { Bouri, Elie } & \text { Galloppo, Giuseppe } & \text { LeVeck, Brad L. } \\ \text { Breitschopf, Barbara } & \text { Garcia-Appendini, Emilia } & \text { Li, Rui } \\ \text { Brown, David } & \text { Glabadanidis, Paskalis } & \text { Lin, Ching-Yi } \\ \text { Brown, Todd } & \text { Golosnoy, Vasyl } & \text { Liu, Hao-Chen } \\ \text { Brusa, Jorge Omar } & \text { Göncü, Ahmet } & \text { Liu, Ruipeng } \\ \text { Buchner, Axel } & \text { Gözgör, Giray } & \text { Marković, Todor } \\ \text { Chang, Chia-Lin } & \text { Guesmi, Khaled } & \text { Marti-Ballester, Carmen Pilar } \\ \text { Cole, Rebel A. } & \text { Hahn, TeWhan } & \text { Mastromatteo, Giuseppe } \\ \text { Crespi, Fabrizio } & \text { Hao, Jia } & \text { Mattos, Fabio } \\ \text { Cumming, Douglas } & \text { Hao, Xuemiao } & \text { McConnell, Patrick } \\ & & \end{array}$


Meles, Antonio

Menoncin, Francesco

Moon, S. Katie

Morley, Bruce

Mourão, Paulo Reis

Mucuk, Mehmet

Mura, Roberto

Narayan, Seema

O'Sullivan, Niall

Okhrin, Ostap

Ouyang, Xiaoling

Overesch, Michael

Panagiotidis, Theodoros

Panapakidis, Ioannis P.

Pancotto, Francesca

Pappas, Vasileios

Payne, James

Polemis, Michael

Porrini, Donatella

Prast, Henriëtte

Ramiah, Vikash
Ratti, Ronald

Reeves, Jonathan J.

Ripplinger, David

Ritter, Matthias

Ryan, Michael

Scelles, Nicolas

Schaumburg, Julia

Schnusenberg, Oliver

Segnon, Mawuli

SenGupta, Indranil

Shimizu, Katsutoshi

Siddiqi, Hammad

Szado, Edward

Tan, Aaron

Tang, Frank

Tannous, George

Teräsvirta, Timo

Theodossiou, Alexandra K.

To, Thuy Duong

Tse, Yiuman

Urzi, Cesira
Vamvoukas, George

Van Leuvensteijn, Michiel

Veliyev, Bezirgen

Viswanath, P.V.

Walter, Terry

Wang, Jessie Jiaxu

Watanabe, Nicholas

Weber, William L.

Westerholm, Joakim

Wielhouwer, Jacco L.

Winkelmann, Lars

$\mathrm{Wu}$, Tsaur-Chin

Wu, Weiping

$\mathrm{Xu}$, Danielle

$\mathrm{Xu}$, Xiaoqing Eleanor

Yan, Meilan

Yang, Jimmy

Yun, Hayong

Zhao, Jessica

(C) 2017 by the author; licensee MDPI, Basel, Switzerland. This article is an open access article distributed under the terms and conditions of the Creative Commons Attribution (CC-BY) license (http://creativecommons.org/licenses/by/4.0/). 